\title{
Efectos de la Depresión en el Bienestar Laboral: El Rol Modulador del Apoyo Social en el Trabajo
}

\section{Effects of depression on worker well-being: The modulator role of work social support}

María Vera ${ }^{1,}{ }^{*}$, Carlos Andrés Vélez ${ }^{1}$, María Fernanda Córdova ${ }^{1}$

\section{INFORMACIÓN DEL}

ARTÍCULO

Fecha de recepción: 24 de noviembre de 2017

Fecha de aceptación: 15 de febrero de 2018

1 Universidad de Especialidades Espíritu Santo, Escuela de Psicología.

\footnotetext{
* Autor de correspondencia: María Vera Perea, Universidad de Especialidades Espíritu Santo. Facultad de artes Liberales y Educación. Escuela de Psicología. Vía La Puntilla $\mathrm{km} 2.5$. E-mail: mveraperea@uees.edu.ec. Tlf: (593-4) 2835630
}

\begin{abstract}
Resumen
Teniendo en cuenta que una de las principales enfermedades laborales es la depresión, tiene sentido estudiar cómo influye esta enfermedad en el bienestar laboral y analizar qué aspectos laborales podrían mejorar la vida laboral a aquellos trabajadores que sufren sus síntomas. Por tanto, el objetivo del presente trabajo es conocer la influencia de la depresión en el bienestar laboral, y analizar el posible efecto modulador del apoyo social en la anterior relación. Mediante análisis de regresión lineal en una muestra de 220 trabajadores se comprobó que la depresión influye negativamente en el bienestar laboral y que el apoyo social posee un rol modulador en la anterior relación, pero no en el sentido esperado. Los resultados obtenidos arrojan interesante información acerca de las prácticas organizacionales en torno a los trabajadores que sufren síntomas depresivos.
\end{abstract}

Palabras Clave:

Apoyo social, Bienestar laboral, Depresión.

Clasificación JEL: I31.

\begin{abstract}
Taking into account that depression is one of the main occupational diseases it makes sense, to study how this disease affects well-being at work and to analyze aspects that could improve employees' working life for those who suffer depression. The objective of this paper is, therefore, to know the influence of depression on labor well-being, and to analyze the possible modulating effect of social support in the relationship. By linear regression analysis in a of a 220 worker sample, it was found that depression negatively influences labor well-being and that social support has a modulating role in the previous relationship, although not in the expected manner. Results provide interesting information concerning organizational practices concerning workers who suffer depressive symptoms.
\end{abstract}

Keywords:

Social support, Well-being, Depression

JEL Classification: I31. 


\section{Introducción}

Las organizaciones han tendido a imponer sus intereses económicos por encima de la salud de los trabajadores, a quienes visualizaban en muchos casos como medios de producción, y a quienes exigían una constante capacitación y actualización profesional para mejorar su desempeño y productividad, soslayando aquella relación afectiva y efectiva que debería existir entre la empresa y la fuerza laboral. Esto se constituye como un enfoque organizacional obsoleto $\mathrm{y}$ perjudicial para la salud del trabajador, sobre todo en el aspecto psicológico, como la depresión y ciertos efectos colaterales que inciden negativamente en su bienestar laboral, que trata de ser fortalecido por medio del apoyo social brindado por la organización (Luthans et al., 2007). Por tanto el objetivo de este trabajo es conocer la influencia de la depresión en el bienestar laboral, y analizar el posible efecto modulador del apoyo social en la anterior relación

El bienestar laboral se entiende como aquel estado en el que los colaboradores perciben y experimentan estímulos $\mathrm{y}$ emociones positivas por cuanto se sienten conformes y contentos con su trabajo y las labores que desempeñan (Valencia et al., 2014). En este sentido, López (2015), acota que el bienestar social contempla programas y beneficios, los cuales están encaminados a abordar aquellas necesidades de los trabajadores que influyen en su comodidad y gusto por su trabajo, y que afianzan su sentido de pertenencia con la empresa de la que forman parte.
Por tanto, el bienestar laboral ha sido abordado dentro de las organizaciones mediante programas con distintos enfoques y objetivos. Dichos objetivos se centran en mejorar las condiciones morales y mentales del trabajador, modificar las condiciones de trabajo, compensación de las condiciones sociales y salariales, estrategias para el incremento de la lealtad y la motivación, $y$ el desarrollo de condiciones que permitan mejorar de manera sustancial la calidad de vida laboral (Barley \& Kunda, 1992). En este estudio, se sigue la línea de trabajo de Blanch et al. (1996) que entiende el bienestar laboral como un constructo formado por las dimensiones: bienestar psicosocial y efectos colaterales.

Por tanto, para entender el bienestar laboral hacen falta planteamientos holísticos y no solamente de manera parcial o con alguna variable psicológica, social o física única (Jiménez \& Cardona, 2014), por tal razón, en esta investigación se abordan dos variables que pueden influir en el bienestar laboral: la depresión y el apoyo social, muchas veces pasados por alto en el paradigma organizacional tradicional, centrado principalmente en la búsqueda, selección y retención de los empleados.

A comienzos del siglo XXI, organizaciones internacionales como la Organización Mundial de la Salud (OMS), alertan del incremento de las enfermedades asociadas al trabajo, que generan no solo estragos físicos sino también diferentes tipos de trastornos psíquicos (Sieglin \& Ramos, 2007). 
Dentro de estas afecciones, surge la depresión como un factor degenerativo que afecta el bienestar laboral, entendida como un estado emocional patológico que se caracteriza por sentimientos exagerados de tristeza, melancolía, abatimiento, disminución de la autoestima, vacío, desesperanza y aislamiento que no siempre responden a una causa real explicable (Fernández-D'Pool et al., 2005).

La depresión y el bienestar laboral tienen impactos únicos e incrementales en la satisfacción de vida e íntimamente asociados con el desempeño laboral (Haslam et al., 2005), pues cuando un individuo atraviesa por una crisis depresiva, su nivel de motivación y efectividad tiende a disminuir, y a su vez su insatisfacción personal aumenta (Salanova et al., 2011), lo que deriva en empleados desmotivados, con problemas de salud psicológica, sin sentido de pertenencia e identidad, todo lo contrario a lo que requieren las empresas hoy en día (Salanova \& Schaufeli, 2004).

La depresión tiende muchas veces a ser estigmatizada e ignorada en el ámbito laboral, no obstante, ésta se asocia con altos grados de discapacidad, y repercute de manera negativa tanto en la productividad individual como en la colectiva, con impacto económico alto en las organizaciones empresariales, sobre todo si se considera que por ejemplo en Europa, alrededor del $86 \%$ de las personas diagnosticadas con depresión pertenecen a la población económicamente activa (Castellón et al., 2016).

Además, la depresión merece especial atención en el campo de la salud pública, puesto que en muchos casos tiende a volverse crónica y presenta episodios de recurrencia con mucha frecuencia (Castellón et al., 2016). Además, Vera (2006), agrega que, independientemente de los factores que generan el estado de depresión en el individuo, su génesis puede entenderse como la presencia de emociones negativas y/o la ausencia de emociones positivas. Esto explica por qué resulta necesario tomar en cuenta el alcance del reforzamiento de aquellos aspectos postivos como un medio terapéutico que favorezca el bienestar general de las personas (Vecina, 2006), lo cual se aplica en todos sus aspectos, incluyendo el laboral.

Dentro de la empresa, uno de los aspectos laborales que podría amortiguar los efectos negativos de la depresión en el bienestar laboral es el apoyo social. Entendido como el soporte brindado por miembros de una misma red social, quienes son emocionalmente significativos y relevantes entre sí, y que aportan al bienestar físico y mental de cada uno de los individuos (Orcasita \& Uribe, 2010).

La mayor fuente de apoyo social proviene de los supervisores y de los mismos trabajadores, y que además, aquellos trabajadores que reciben el apoyo social muestran una mayor productividad y mayor bienestar psicológico. Además, este tipo de apoyo social puede ser percibido y recibido mediante la colaboración en las tareas realizadas en el trabajo, o mediante el apoyo emocional que se identifica con la empatía y consejería (Pérez \& Alcover, 2011). 
Haslam et al. (2004) evidenciaron los efectos negativos de la depresión en el desempeño laboral. Por otra parte, Mahan et al. (2010) demostraron que el apoyo social por parte de los compañeros de trabajo disminuye los niveles de ansiedad y depresión. Sin embargo, el apoyo social no funcionó como un modulador entre los estresores laborales y la depresión. En esta línea, Park (2002) concluyó que el apoyo corporativo es mucho más eficiente que el apoyo social prestado por supervisores y compañeros de trabajo al momento de modular los efectos negativos de la depresión. No obstante, el estudio concluye también que el apoyo social brindado por parte de los compañeros, supervisores e incluso gerentes, es en muchos casos importante para mitigar los riesgos de depresión en la fuerza laboral. Por último, un estudio llevado a cabo por Safe Work Australia (2013), mostró que aquellos trabajadores que mostraban bajo apoyo por parte de compañeros, supervisores y gerentes, presentaron más del doble de síntomas depresivos que aquellos que manifestaron tener un nivel alto de apoyo.

Por todo lo anterior, las hipótesis del presente trabajo son:

$H_{l a}:$ La depresión se relaciona negativa y significativamente con el bienestar psicosocial. Es decir, aquellos trabajadores con alta sintomatología en depresión presentarán menores puntuaciones en bienestar psicosocial.

$H_{l b}$ : La depresión se relaciona positiva significativamente con los efectos colaterales. Es decir, aquellos trabajadores con alta sintomatología en depresión presentarán mayores puntuaciones en efectos colaterales.

$H_{2 a}:$ El Apoyo Social modula la relación entre depresión y bienestar psicosocial. De manera que el efecto negativo de la depresión en el bienestar psicosocial será menor en aquellos trabajadores que perciban un alto apoyo social.

$H_{2 b}$ : El Apoyo Social modula la relación entre depresión $\mathrm{y}$ efectos colaterales. De manera que el efecto potenciador de la depresión en los efectos colaterales será menor en aquellos trabajadores que perciban un alto apoyo social.

\section{Materiales y métodos}

\section{Muestra y procedimiento}

La muestra consiste en 220 trabajadores. Dichos trabajadores son el $100 \%$ de la plantilla de una empresa elegida por conveniencia del sector de maquinaria pesada en Ecuador. En dicha muestra participa tanto el personal operativo como el administrativo, teniendo en cuenta a gerentes, directivos, jefes, coordinadores, analistas y técnicos mecánicos. El 64,5\% de la población son hombres y $35,5 \%$ son mujeres. Además, el $61 \%$ de la muestra se encuentra entre los 19 y 35 años de edad; el $25 \%$ entre 36 y 49 años; $14 \%$ entre 50 y 68 años. En cuanto al procedimiento, se envió un correo a todo el personal de la empresa adjuntando el link con cuestionario e instrucciones. Además, en dicho correo se aseguraba el anonimato y confidencialidad 
a todos los participantes. Los supervisores de la empresa animaron a todos los trabajadores a contestar dicho cuestionario.

\section{Instrumentos}

Bienestar laboral: Se utilizó el Cuestionario de Bienestar Laboral General de Blanch et al. (1996). El cuestionario consta de dos dimensiones:

1) Bienestar psicosocial, y

2) Efectos colaterales.

La dimensión de bienestar psicosocial consta de las siguientes escalas: afectos, competencias y expectativas ("En mi trayectoria laboral, mi motivación por el trabajo es"). La escala de respuesta es tipo Likert de 7 puntos, donde 1 es la puntuación más baja y 7 la más alta. Por otra parte, la dimensión de efectos colaterales consta de las siguientes escalas: somatización, desgaste $\mathrm{y}$ alienación. ("Actualmente por causa de mi trabajo siento dolores de cabeza"). La escala de respuesta utilizada es tipo Likert de 7 puntos, donde 1 es la puntuación más baja y 7 la más alta. Alpha de Cronbach $=$ 0,81 .

Depresión: Se utilizó el Inventario de Depresión de Beck et al. (1996), el cual contiene 21 ítems ("Me siento triste todo el tiempo y no puedo liberarme de ello"). Las respuestas son de selección múltiple, donde se otorgan puntuaciones que van de cero a tres siendo siempre la primera pregunta cero y tres el último enunciado de cada pregunta, la escala posee un coeficiente alfa de Cronbach de 0,83.
Apoyo social: Se utilizó el Cuestionario Psicosocial de Copenhague (COPSOQ) del Instituto Nacional de Seguridad y Salud Laboral de Dinamarca (AMI). En la validación española ISTAS 21 (2003). Se utilizó los ítems 13, 14 y 18, correspondientes al apoyo social de compañeros y supervisores ("Entre compañeros y compañeras se ayudan en el trabajo"). El tipo de respuesta es tipo Liket de 5 puntos, donde 1 es nunca y 5 es siempre.

\section{Resultados}

En primer lugar, se realizaron los análisis descriptivos de las variables del estudio. En la Tabla 1 se pueden ver medias, desviaciones típicas, alpha de Cronbach y correlaciones de las variables. Los alphas son aceptables y las correlaciones son significativas en todos los casos y muestran el signo esperado, a saber: la relación entre depresión y bienestar psicosocial es negativa, lo que significa que a mayor depresión, menor bienestar.

La relación entre depresión y efectos colaterales es positiva, lo que indica que a mayor depresión, mayor percepción de efectos colaterales. Entre depresión y apoyo social es negativa, es decir, a mayor depresión, menos percepción de apoyo social. Entre bienestar psicosocial y efectos colaterales es negativa, es decir, a mejor bienestar, menor número de efectos colaterales percibidos. Entre bienestar psicosocial y apoyo social, positiva, a más de la primera, más de la segunda y por último entre efectos colaterales y apoyo social es negativa, a mayores efectos. 
Tabla 1.

Análisis descriptivos $(\mathrm{N}=220)$

\begin{tabular}{lccccccc}
\cline { 2 - 8 } & $\bar{X}$ & $S D$ & $\alpha$ & 1 & 2 & 3 & 4 \\
\hline Depresión & 6,54 & 8,12 & 0,91 & 1 & $-0,56^{* * *}$ & $0,42^{* * * *}$ & $-0,34^{* * *}$ \\
Bienestar psicosocial & 5,92 & 1,12 & 0,88 & 1 & $-0,61^{* * *}$ & $0,56^{* * *}$ \\
Efectos colaterales & 2,84 & 1,54 & 0,87 & & 1 & $-0,41^{* *}$ \\
Apoyo social & 3,82 & 0,92 & 0,65 & & & 1 \\
\hline *** $p<0.001$ & & & & & &
\end{tabular}

En segundo lugar, se llevó a cabo una regresión lineal por pasos con el paquete estadístico IBM SPSS. El primer modelo es el que tiene el bienestar psicosocial como variable dependiente, en dicho modelo, los resultados son los siguientes: 1) Primer paso: incluir la depresión como predictor del bienestar psicosocial, obteniendo $\beta=$ $-0,56 p=0,00$. Es decir, se confirma la $H_{1 a}$ : la depresión se relaciona negativamente con el bienestar psicosocial; 2) Segundo paso: incluir el apoyo social como predictor del bienestar, teniendo en cuenta el efecto de la depresión, obteniendo $\beta=0,42 ; p=$ 0.00 . Demostrando que el apoyo social es importante para el bienestar; y 3) Tercer paso: se realizó la interacción de apoyo social y bienestar, poniendo a prueba la $H_{2 a}$ : el apoyo social modula la relación entre depresión y bienestar psicosocial. Se obtuvo $\beta=-0,36 ; p=0,05$, confirmando la $H_{2 a}$. El modelo final, con todos los predictores explica un $47 \%\left(R^{2}\right.$ ajustado $=$ $0,47)$ de la varianza de la variable bienestar psicosocial.

El segundo modelo es el que tienen los efectos colaterales como variable dependiente. En dicho modelo, los resultados son los siguientes: 1) Primer paso: incluir la depresión como predictor de los efectos colaterales, obteniendo $\beta=$ 0,$42 ; p=0,00$. Es decir, se confirma la $H_{l b}$ : La depresión se relaciona positivamente con los efectos colaterales;
2) Segundo paso: incluir el apoyo social como predictor de los efectos colaterales, teniendo en cuenta el efecto de la depresión, obteniendo $\beta=-0,30 ; p=0,00$. Demostrando que el apoyo social es importante para los efectos colaterales, y 3) Tercer paso: la interacción de apoyo social y efectos colaterales, poniendo a prueba la $H_{2 b}$ : el apoyo social modula la relación entre depresión $\mathrm{y}$ efectos colaterales. Se obtuvo $\beta=0,65 p=0,003$, confirmando la $H_{2 b}$. El modelo final, con todos los predictores explica un $28 \%$ de la varianza de los efectos colaterales $\left(R^{2}\right.$ ajustado $=0,28)$. En las figuras 1 y 2 se puede apreciar el efecto modulador del apoyo social tanto en el bienestar psicosocial como en los efectos colaterales.

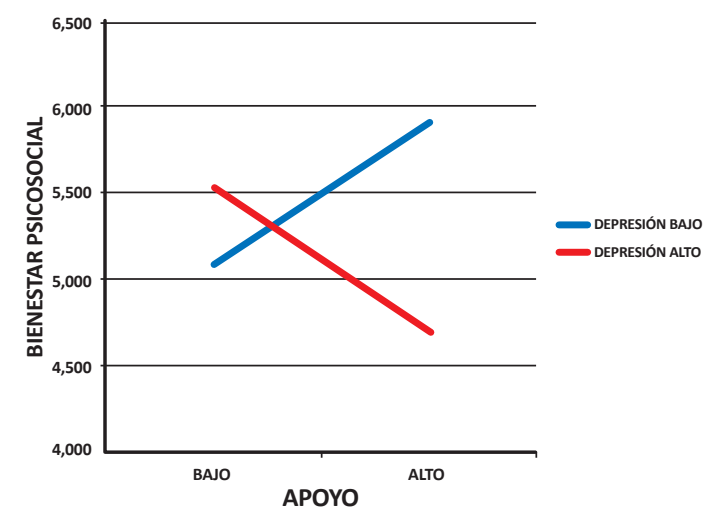

Figura 1. Modulación en bienestar psicosocial.

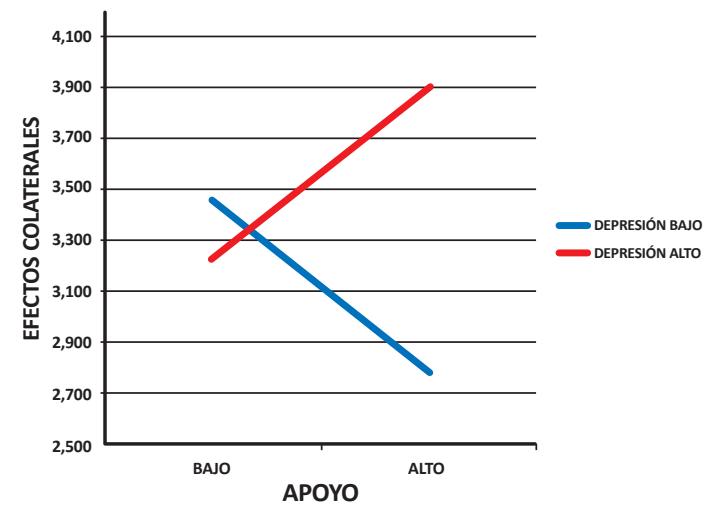

Figura 2. Modulación en efectos colaterales. 


\section{Discusión}

El objetivo de la presente investigación ha sido analizar el efecto de la depresión en el bienestar laboral (bienestar psicosocial y efectos colaterales), prestando especial atención al efecto modulador que tiene el apoyo social sobre la anterior relación. Se han confirmado todas las hipótesis del estudio, por tanto, se puede afirmar que:

1) La depresión tiene un efecto negativo y significativo sobre el bienestar laboral (en ambas dimensiones), y a pesar de que la muestra presenta niveles bajos de depresión, se confirma que el bienestar de los trabajadores disminuye a medida que se incrementan los cuadros depresivos;

2) Se ha confirmado el efecto modulador del apoyo social sobre la relación entre depresión y el bienestar laboral (en sus dos dimensiones). Sin embargo, los resultados no son como se esperaban. La literatura previa (Mahan et al., 2010; Park, 2002) muestra el apoyo social siempre como una ayuda, como un facilitador, y por lo tanto, se esperaba que el apoyo social fuera un factor que disminuyera los efectos negativos que provoca la depresión sobre el bienestar. Tal y como se puede apreciar en las dos figuras, el apoyo social actúa de dos maneras diferentes dependiendo de si los trabajadores muestran alta o baja depresión. En el caso de los trabajadores que perciben una baja depresión, el apoyo social actúa conforme a lo esperado, es decir, hace que la relación entre depresión y bienestar se vuelva menos fuerte, y por tanto hace que los efectos en la depresión no reduzcan el bienestar de los trabajadores. Sin embargo, en los trabajadores con alta percepción de depresión, la relación funciona de manera muy diferente, el apoyo social no solo no ayuda, sino que empeora el bienestar laboral. Parece que en los trabajadores que muestran síntomas depresivos, el apoyo social no es una solución para que se sientan mejor en el trabajo. Esto podría deberse a la relación tan fuerte que existe entre el aislamiento social y la depresión (Thoits, 1995; Underwood, 2000). De esta manera, parece que aquellos empleados que muestran mayores síntomas de depresión no desean el apoyo brindado por compañeros y jefe, sino permanecer aislados en la empresa.

\section{Implicaciones teóricas y prácticas}

En cuanto a las implicaciones teóricas, la presente investigación aporta nueva información sobre el rol de uno de los recursos más utilizados dentro de la Psicología organizacional: el apoyo social. Mostrando, en contra de la literatura existente, una situación muy concreta (empleados con sintomatología depresiva) un efecto negativo sobre el bienestar laboral.

En cuanto a las implicaciones prácticas, existe un significativo aporte a la gestión que desarrollan los profesionales de Recursos Humanos pues se debe analizar a profundidad la salud mental de los trabajadores antes de poner en práctica una decisión organizacional de cualquier índole, pues a las personas deprimidas este "aporte" les causa efectos perjudiciales en su salud mental y por ende en su desempeño laboral. En estos caso, lo que pareciera conveniente es ayudar a los 
trabajadores con su sintomatología y luego proporcionarles el apoyo social de compañeros y supervisores.

\section{Fortalezas y limitaciones}

En cuanto a las fortalezas de este estudio, se puede destacar, la población elegida para realizar la investigación. Por un lado, ha participado el $100 \%$ de la empresa, y por otro lado, se dedican a un sector de la población muy poco estudiada hasta ahora: la maquinaria pesada en el Ecuador. Sin embargo, esto también conlleva limitaciones, ya que al ser una muestra enfocada en un solo sector, resulta difícil asumir que el mismo modelo puede responder las interrogantes en todos los sectores empresariales.

Quizás, en estos otros sectores existan niveles de depresión mucho más altos, y en los que las condiciones laborales y las políticas empresariales limitan los programas de apoyo social, dejando una percepción mucho más negativa en los trabajadores que las que se tiene en la muestra analizada. En esos casos, el bienestar laboral se vería más duramente afectado. Por lo tanto, es importante que se efectúen futuras investigaciones en las que se analicen otros escenarios laborales, en los que las percepciones sean distintas, y cuyos datos sean contrastados con los obtenidos en estudios como el aquí presente.

Como conclusión, teniendo en cuenta que la OMS ha alertado sobre el incremento de las enfermedades asociadas al trabajo, como la depresión, que generan no solo estragos físicos sino también diferentes tipos de trastornos psíquicos (Sieglin \& Ramos, 2007), es importante conocer cómo ayudar a los empleados con dicha sintomatología.

Este estudio ha comprobado que la depresión empeora el bienestar laboral y que el apoyo social solo ayuda cuando la sintomatología es baja.

\section{Referencias}

Barley, S. R. \& Kunda, G. (1992). Design and devotion: Surges of rational and normative ideologies of control in managerial discourse. Administrative Science Quarterly, 37, 363-399.

Beck, A. T., Steer, R. A., \& Brown, G. K. (1996). Manual for the Beck Depression Inventory-II. San Antonio, TX: Psychological Corporation.

Blanch, J. M., Sahagún, M., Cantera, L., \& Cervantes, G. (1996). Cuestionario de Bienestar Laboral General: Estructura y Propiedades Psicométricas. Revista de Psicología del Trabajo y de las Organizaciones, 26, 157-170.

Castellón, E., Ibern, P., Gili, M., Lahera, G., Sanz, J., \& Saiz, J. (2016). El abordaje de la depresión en el ámbito del trabajo: recomendaciones clave. Psiquiatría Biológica, 23, 112-117.

Fernández-D'Pool, J., Fernández, P., \& Acevedo, J. (2005). Estrés organizacional, depresión y afrontamiento en trabajadores petroleros. Salud de los Trabajadores, 13, 7-18.

Haslam, C., Atkinson, S., Brown, S., \& Haslam, R. (2005). Anxiety and depression in the workplace: Effects on the individual and the organisation (a focus group investigation). Journal of Affective Disorders, 88, 209-215. 
ISTAS-COPSOQ Navarra (2003). Encuesta del ambiente psicosocial de trabajo en la Comunidad Autónoma de Navarra. ISTAS: Barcelona 2003.

Jiménez, A. \& Cardona, L. (2014). Guía para el manejo de los colaboradores diagnosticados con trastorno de depresión mayor: Una propuesta desde el enfoque biopsicosocial con aplicabilidad a las organizaciones. Recuperado de: http://bibliotecadigital.usb.edu.co/bitstream/ 10819/2279/1/Guia_Colaboradores_Depres ion_Jimenez_2014.pdff

Mahan, P., Mahan, M., Park, N. J., Shelton, C., Brown, K., \& Weaver, M. (2010). Work Environment Stressors, Social Support, Anxiety, and Depression Among Secondary School Teachers. AAOHN Journal, 58, 197-205.

López, S. (enero de 2015). Bienestar laboral en los trabajadores de una empresa portuaria de Escuintla. Recuperado de:

http://recursosbiblio.url.edu.gt/tesisjcem/2 015/05/43/Lopez-Shelvy.pdf

Luthans, F., Avolio, B., Avey, J., \& Norman, S. (2007). Positive Psychological Capital: Measurement and Relationship with Performance and Satisfaction. Personnel Psychology, 60, 541-542.

Orcasita, L. \& Uribe, A. (2010). La importancia del apoyo social en el bienestar de los adolescentes. Psychologia: Avances de la disciplina, 4, 69-82.

Park, K. O. (2002). The effects of social support at work on job demands, job control, depression, job performance and absenteeism. Recuperado de: https://getd.libs.uga.edu/pdfs/park_kyoun g_o_200208_phd.pdf

Pérez, V. \& Alcover, C. (2011). Apoyo social, satisfacción laboral y abandono en trabajadores con discapacidad. Boletín de Psicología, 102, 23-41.
Safe Work Australia (2013). The relationship between work characteristics, wellbeing, depression and workplace bullying. Recuperado de: https://www.safeworkaustralia.gov.au/syste $\mathrm{m} /$ files/documents/1702/wellbeing-depressi on-bullying-technical-findings.pdf

Salanova, M., Lorente, L., Chambel, M., \& Martínez, I. (2011). Linking transformational leadership to nurses' extra-role performance: The mediating role of self-efficacy and work engagement. Journal of Advanced Nursing, 67, 2256-2266.

Salanova, M. \& Schaufeli, W. (2004). El engagement de los empleados: un reto emergente para la Dirección de los Recursos Humanos. Revista de trabajo y seguridad social, 2, 109-138.

Sieglin, V. \& Ramos, M. (2007). Estrés laboral y depresión entre maestros del área metropolitana de Monterrey. Revista Mexicana de Sociología, 69, 517-551.

Thoits, P. A. (1995). Stress, coping, and social support processes: where are we? What next? Journal of Health Social Behavior, Extra issue, 53-79.

Underwood, P. W. (2000). Social support. The promise and reality. In B. H., Rice, (Ed.). Handbook of stress. Sage: Los Angeles.

Valencia, J., García, C., Alvarán, M., \& Sánchez, D. (2014). Diagnóstico de las causas que influyen en el bajo nivel de satisfacción y sensación de infelicidad percibida por las personas al interior de las organizaciones. Recuperado de:

http://repository.udem.edu.co/handle/114 07/1258

Vecina, M. (2006). Emociones Positivas. Papeles del Psicólogo, 27, 9-17.

Vera, B. (2006). Psicología Positiva: Una nueva forma de entender la psicología. Papeles del Psicólogo, 27, 3-8. 